\title{
Résultats pour la santé en ce qui concerne les enfants à faible revenu souffrant d'asthme actif au Canada
}

\author{
TTo, Ph.D. (1, 2); S Dell, M.D. (1,3); M Tassoudji, M.Sc. (1); C Wang, M.D. (1)
}

\section{Résumé}

Les données de l'Enquête longitudinale nationale sur les enfants et les jeunes du Canada recueillies en 1994-1995 et en 1996-1997 ont servi à mesurer les résultats longitudinaux pour la santé chez les enfants asthmatiques. Plus de 10000 enfants âgés de 1 à 11 ans à l'égard desquels existaient des données complètes sur leur état asthmatique pour les deux années ont été inclus. Les résultats englobaient les hospitalisations et l'utilisation des services de santé. Les enfants souffrant d'asthme actif étaient des enfants qui avaient reçu un diagnostic d'asthme de la part d'un médecin et qui prenaient régulièrement des médicaments à inhaler, avaient eu une respiration sifflante ou une crise au cours de l'année précédente ou devaient limiter leurs activités en raison de l'asthme. Le fait d'avoir de l'asthme faisait augmenter de façon importante les risques d'hospitalisation $(\mathrm{RC}=2,52$; IC de $95 \%: 1,71,3,70)$ et l'utilisation des services de santé $(\mathrm{RC}=3,80$; IC $95 \%: 2,69,5,37)$. La faible adéquation du revenu en 1994-1995 est un prédicteur de significatif de l'hospitalisation et à de l'utilisation des services de santé en 1996-1997 ( $\mathrm{RC}=2,68$; IC de $95 \%: 1,29,5,59$ et $\mathrm{RC}=0,67$; IC de $95 \%: 0,45,0,99$ respectivement). Nos résultats confirment que le fait de souffrir d'asthme actif et de vivre au sein d'une famille à faible revenu avait une incidence importante sur l'état de santé des enfants du Canada. Les programmes visant à réduire le coût économique des hospitalisations en pédiatrie devraient être axés sur les personnes asthmatiques et à faible revenu.

Mots clés : asthme, faible revenu, hospitalisation, utilisation des services de santé

\section{Introduction}

L'asthme est considéré comme le motif le plus fréquent d'hospitalisation évitable chez les enfants ${ }^{1-4}$. Il est généralement convenu que l'asthme crée des « conditions propices aux soins ambulatoires » c.-à-d. qu'une bonne gestion des patients externes devrait permettre de réduire les hospitalisations ${ }^{5}$. Une maîtrise inadéquate de l'asthme peut s'avérer coûteuse; cette maladie a des conséquences physiques et peut diminuer la qualité de vie d'une personne. En 1998, on estimait que les coûts directs principaux des soins de santé liés à l'asthme (hospitalisations et dépenses liées aux médicaments) totalisaient près de 402 millions de dollars ${ }^{6}$. Selon un rapport de l'Institut de recherche en services de santé (To et coll.), les coûts totaux annuels directs et indirects par enfant canadien asthmatique se chiffraient à 676 \$ en $1995^{7}$. Les hospitalisations représentaient la part la plus importante des coûts totaux, $77 \%$. Les autres utilisations des services de santé (c.-à-d. les visites chez l'omnipraticien et chez le spécialiste, les consultations et les services d'urgence) constituaient $21 \%$ des coûts totaux ${ }^{8}$.

La plupart des données sur l'utilisation des services de santé et les résultats cliniques chez les enfants asthmatiques sont tirées d'enquêtes transversales ou de dossiers administratifs ${ }^{9,10}$. À notre connaissance, aucune enquête longitudinale systématique de la population n'a été menée pour déterminer la prévalence de l'asthme au sein de divers groupes d'âge, le taux d'utilisation des services de santé (USS) et la morbidité associée à l'asthme. L'Enquête longitudinale nationale sur les enfants et les jeunes (ELNEJ), enquête longitudinale de la population canadienne, constitue un excellent moyen d'étudier l'asthme chez les enfants de façon prospective et d'obtenir des estimations stables de la prévalence de l'asthme. Plus précisément, l'état asthmatique qui est évalué de façon longitudinale procure un moyen de différencier les asthmes "persistants» des asthmes "épisodiques » et d'évaluer de façon prospective l'incidence des facteurs présents pendant les années préscolaires sur les résultats des enfants d'âge scolaire. L'objectif de la présente étude comporte trois volets : 1) créer une cohorte longitudinale d'une population d'enfants canadiens atteints d'asthme actif, 2) mesurer l'USS de ces enfants et leurs résultats cliniques par rapport à ceux des enfants qui ne sont pas atteints d'asthme, 3) évaluer l'incidence de l'asthme sur l'USS et les résultats cliniques chez les enfants en tenant compte d'autres facteurs de risque.

\section{Méthodes \\ Source des données}

Les données longitudinales du cycle 1 (C1) de l'ELNEJ de 1994-1995 et du cycle 2 (C2) de 1996-1997 ont été utilisées pour notre étude. L'ELNEJ est une enquête prospective

Coordonnées des auteurs

1 Sciences de l'évaluation de la santé des enfants, Institut de recherche, Hospital for Sick Children, Toronto (Ontario).

2 Département des sciences de la santé publique, Université de Toronto, Toronto (Ontario).

3 Division de la médecine respiratoire, Hospital for Sick Children; Faculté de médecine, Université de Toronto, Toronto (Ontario).

Correspondance : $D^{\text {re }}$ Teresa To, Sciences de l'évaluation de la santé des enfants, Institut de recherche, Hospital for Sick Children, 555 avenue University, Toronto (Ontario) M5G 1X8, Tél.: 416-813-8498, Téléc.: 416-813-5979, Courriel : teresa.to@sickkids.ca 
longitudinale conçue pour mesurer l'état de santé et le développement de l'enfant ${ }^{11,12}$. Elle recourt à un échantillonnage en grappes stratifié à plusieurs degrés de sorte que l'échantillon de l'étude soit représentatif de la population d'enfants canadiens. Les grappes ont été constituées de manière à fournir des échantillons suffisants dans les grandes zones géographiques et dans sept groupes d'âge clés. Les intervieweurs de Statistique Canada se sont rendus dans les ménages et y ont remis des questionnaires standardisés à la personne qui connaît le mieux (PCM) l'enfant, laquelle était la mère biologique dans $89,9 \%$ des cas. Le taux de réponse global à l'enquête a été de $86,4 \%$ pour le $\mathrm{C} 1$ et de $91,6 \%$ pour le $\mathrm{C} 2$. On a obtenu le consentement éclairé des tuteurs légaux ou de l'enfant selon le cas. La description complète de l'ELNEJ se trouve à l'adresse (http:www.statcan.gc.ca/ bsolc/olc-cel/olc-cel? catno $=89$ F0078XIF \&lang $=$ fra) ${ }^{11}$.

L'ELNEJ comprend tous les enfants échantillonnés dans le $\mathrm{C} 1$ qui faisaient partie des ménages qui ont répondu à l'enquête. Même si certains des enfants qui ont participé au $\mathrm{C} 1$ n'ont pas participé au $\mathrm{C} 2$ pour diverses raisons (p. ex. réduction volontaire afin de diminuer la taille de l'échantillon, suivi impossible, ou défaut de réponse à l'enquête de la part de la mère ou du père biologique), nous nous sommes efforcés de limiter le nombre de ces enfants le plus possible afin de pouvoir effectuer une recherche longitudinale par cohorte d'âge à l'échelle nationale ${ }^{12}$. Pour la présente étude, nous avons inclus au départ dans les analyses 10148 enfants (échantillon pondéré de 3128 645) de 1 à 11 ans $(\mathrm{C} 1)$ dont la mère ou le père biologique avait répondu à l'enquête et pour l'état asthmatique desquels existaient des données complètes dans les deux cycles.

\section{Définition de l'asthme actif}

Le nombre des cas d'asthme actif était validés lorsque la PCM faisait état d'un diagnostic d'asthme posé par un professionnel de la santé au sujet de son enfant et d'au moins une des situations suivantes : 1) l'enfant prenait régulièrement des médicaments sur ordonnance à inhaler, 2) l'enfant avait eu une respiration sifflante ou une crise d'asthme dans les 12 mois précédents, ou 3) l'asthme de l'enfant l'empêchait de participer à des activités scolaires ou à d'autres activités normales ou limitait sa participation à ces activités ${ }^{13}$. L'état asthmatique a été subdivisé en quatre catégories : 1) "sans asthme " (pas d'asthme actif dans les deux cycles), 2) "nouveau cas d'asthme " (asthme actif dans le C2 seulement), 3) " asthme épisodique» (asthme actif dans le $\mathrm{C} 1$ seulement), et 4) "asthme persistant » (asthme actif dans les deux cycles). Seuls les enfants qui avaient été classés dans les catégorisés "asthme persistant» (AP) ou "sans asthme» (SA) (total de 9462 cas ou échantillon pondéré de 2908 136) ont été inclus dans notre analyse longitudinale. Dans l'analyse longitudinale multivariable, les résultats cliniques de chaque enfant au fil du temps ont été examinés à l'aide du modèle de l'équation d'estimation généralisée (EEG) $)^{14-17}$ qui intègre l'état asthmatique actif aux deux cycles tout en tenant compte de la corrélation intra-individus.

\section{Mesure des résultats}

Les résultats des traitements ont été déterminés à la lumière des déclarations des parents sur l'hospitalisation de leur enfant et leur USS dans les deux cycles (C1 et $\mathrm{C} 2$ ). L'hospitalisation a été définie comme un séjour d'une nuit à l'hôpital au cours des douze derniers mois. L'USS a été définie comme l'aide ou les conseils que les parents cherchent à obtenir auprès d'un médecin, et elle a été déterminée selon la réponse de la PCM à la question : "L'an dernier, combien de fois avez-vous vu un médecin ou parlé avec lui au sujet de la santé de votre enfant? ". L'hospitalisation et l'USS ont été codées comme des variables dichotomiques (oui/non) afin de réduire au minimum le biais de rappel.

\section{Facteurs de risque}

Les autres variables indépendantes envisagées à titre de facteur de confusion possible étaient les suivantes : sexe, âge, état de santé de la mère et de l'enfant, troubles chroniques de l'enfant, antécédents de respiration sifflante chez l'enfant, tabagisme parental, antécédents maternels d'asthme, symptômes maternels de dépression (n'ayant pas fait l'objet d'un examen antérieur en regard de l'hospitalisation et de l'utilisation de services de santé chez les asthmatiques), indicateurs de statut socioéconomique (SSE) et statut d'immigrant de la mère.

L'état de santé de l'enfant a été déterminé d'après l'évaluation de la santé de l'enfant par sa mère (excellente/très bonne ou bonne/satisfaisante/mauvaise). L'état de santé de l'enfant était consigné si la PCM avait fait mention d'un diagnostic d'allergies ou de bronchite posé par un médecin. Une respiration bruyante ou sifflante observée à tout moment au cours des 12 derniers mois caractérisaient l'enfant comme ayant une respiration sifflante.

L'état de santé de la mère a été déterminé d'après une autoévaluation (excellente/très bonne ou bonne/satisfaisante/mauvaise). Le tabagisme parental a été établi en fonction d'une consommation de cigarettes quotidienne, occasionnelle ou nulle.

Pour l'adéquation du revenu, les répondants ont été subdivisés en groupes à faible revenu, à revenu moyen et à revenu élevé en fonction du revenu total du ménage et du nombre de membres du ménage ${ }^{11,18}$. La mère d'un enfant a été considérée comme une immigrante si elle a déclaré avoir immigré au Canada. L’âge de l'enfant a été mesuré par année et utilisé comme une variable continue.

\section{Analyse statistique}

Le modèle de l’Équation d'estimation généralisée (EEG) ${ }^{14-17}$ pour les données longitudinales catégoriques a servi à intégrer les covariables " chronologiques " dans la modélisation des prédicteurs de l'USS ou de l'hospitalisation. L'EEG a servi à tenir compte des effets longitudinaux des prédicteurs sur l'USS ou l'hospitalisation dans le $\mathrm{C} 1$ ou le $\mathrm{C} 2$, et les données su les prédicteurs des deux cycles à mesurer la probabilité globale ajustée de l'USS ou de l'hospitalisation. Les effets résiduels (c.-à-d. l'effet d'un facteur de risque dans le C1 sur l'USS ou l'hospitalisation dans le C2) ont également été pris en considération.

Les guides de Statistique Canada ${ }^{11,12}$ sur la publication des données ont été observées, et les poids d'échantillonnage longitudinaux calculés par Statistique Canada ${ }^{11,12}$ ont été appliqués à l'analyse des caractéristiques 
de la population étudiée de sorte que les estimations qui en découlent puissent être considérées comme représentatives de la population totale des enfants de 1 à 11 ans au départ. Les coefficients de variation calculés par Statistique Canada ${ }^{11}$ ont servi à déterminer la qualité des estimations. En conséquence, les estimations qui ne répondaient pas aux critères de Statistique Canada ont été signalés.

Pour les analyses à variables multiples, les poids d'échantillonnage standardisés ont été utilisés afin de préserver la taille initiale de l'échantillon, pour ainsi éviter une surestimation de la signification statistique tout en conservant les mêmes distributions que celles obtenues à l'aide des poids de la population ${ }^{19}$. Seules les variables statistiquement significatives $(p<0,05)$ ou les facteurs de confusion connus tels que le sexe, l'âge, la faible adéquation du revenu (FAR) et le statut d'immigrant de la mère ont été inclus dans les modèles finaux ${ }^{20}$. Les corrélations entre les covariables ont été examinées, et la trousse statistique du SAS (version 8.0 du SAS, Cary, Caroline du Nord, États-Unis) a servi à toutes les analyses ${ }^{21}$.

\section{Résultats}

\section{Caractéristiques démographiques}

Le tableau 1 indique la prévalence générale de l'asthme et la prévalence de l'hospitalisation et de l'USS selon les quatre catégories d'état asthmatique. La prévalence générale des cas d'asthme déclarés était de $8,4 \%$ pour le cycle 1 et de $9,4 \%$ pour le cycle 2 . Selon notre classification de l'asthme précédemment définie, $4 \%$ des enfants nouvellement atteints d'asthme, $3 \%$ souffraient d'asthme épisodique et 5,3\%, d'asthme persistant (AP). Globalement, 87,6 \% des enfants ne souffraient pas d'asthme actif (SA) dans les deux cycles. La prévalence de l'hospitalisation était la plus élevée chez les enfants atteints d'AP dans les deux cycles $(16,8 \%$ et $8,3 \%)$ et la plus faible chez les enfants SA. En comparaison des enfants SA, le taux d'hospitalisation était quatre fois plus élevé chez les enfants souffrant d'AP $(16,8 \%$ c. $3,8 \% ; p<0,0001)$. Leur USS était aussi plus élevé $(95,3 \%$ c. $80,3 \%$; $p<0,0001)$.
Le tableau 2 compare des caractéristiques initiales entre les enfants SA et les enfants AP. Globalement, chez les enfants AP, le pourcentage de garçons âgés de 1 à 11 ans était plus élevé que chez les filles $(62,6 \%$ c. $37,4 \% ; p<0,001)$. Les enfants âgés de 9 à 11 ans avaient la plus forte prévalence d'AP en comparaison des enfants âgés de 1 à 4 ans et de 5 à 8 ans $(6,8 \%$ c. $4,2 \%$; $p<0,001$ et $6,8 \%$ c. $6,6 \% ; p<0,001)$. Au départ, les enfants SA avaient un meilleur état de santé déclaré que les enfants AP (91,8 \% c. $61 \% ; p<0,001)$. Les enfants AP ont été hospitalisés $(16,8 \%$ c. 3,8 \%; $p<0,001)$ et ont utilisé les services de santé $(95,3 \%$ c. $80,3 \% ; p<0,001)$ en plus grand nombre que les enfants SA. De plus, les enfants AP étaient plus nombreux à souffrir d'allergies $(45 \%$ c. $11,8 \%$; $p<0,001$ ), et le groupe de leurs mères comptait moins d'immigrantes $(13,9 \%$ c. $18,8 \% ; p<0,001)$.

\section{Estimations de l'utilisation des services de santé et de l'hospitalisation}

Notre modèle de régression au tableau 3 montrait que le fait d'être plus jeune et de souffrir d'asthme actif $(\mathrm{RC}=2,52$; IC de $95 \%: 1,71,3,70)$ ou d'avoir une santé actuelle moins qu'excellente ou très bonne $(\mathrm{RC}=3,10$; IC de $95 \%: 2,38$, 4,03 ) accroissait le risque d'hospitalisation. Cependant, le fait d'avoir une mère immigrante $(\mathrm{RC}=0,65$; IC de $95 \%: 0,43$, 0,98 ) diminuait le risque d'hospitalisation. Par ailleurs, le fait d'être plus jeune, d'être atteint d'asthme ( $\mathrm{RC}=3,80$; IC de $95 \%: 2,69,5,37)$, de souffrir d'allergies $(\mathrm{RC}=1,61$; IC de $95 \%: 1,33,1,94)$ et de ne pas être en très bonne ou en excellente santé $(\mathrm{RC}=1,56$; IC de $95 \%: 1,27,1,92)$ donnait lieu à un risque accru d'USS.

Les effets résiduels des covariables ont été examinés. De toutes les covariables étudiées, la faible adéquation du revenu (FAR) avait un effet résiduel statistiquement significatif sur l'hospitalisation et l'USS $(\mathrm{RC}=2,68$; IC de $95 \%: 1,29,5,59$ et $\mathrm{RC}=0,67$; IC de $95 \%: 0,45,0,99)$. Cela signifie que la FAR mesurée au départ avait des effets durables sur l'hospitalisation et l'USS au C2. On a aussi étudié les analyses de régression logistique transversales en utilisant les mêmes facteurs de risque du point de départ et on a obtenu des résultats similaires (non présentés).

\section{Discussion}

Les résultats obtenus de cette étude longitudinale de la population permet de quantifier l'ampleur des hospitalisations et de l'USS chez les enfants atteints d'asthme actif. Nos constatations soutiennent l'hypothèse selon laquelle l'asthme infantile a une incidence importante sur les risques d'USS et d'hospitalisation toutes causes confondues. Peu d'études antérieures ont porté sur ces liens de causalité chez les enfants. Chen et coll. ont souligné que, chez les Canadiens de plus de 12 ans, l'asthme représentait un important facteur de risque général d'hospitalisation, et que le rapport de cotes pour l'asthme fluctuait selon les variables sociodémographiques telles que l'âge et le revenu du ménage ${ }^{22}$. Ces conclusions étaient fondées sur des données transversales de l'enquête et ne tenaient pas compte des modifications possibles de l'état asthmatique au fil du temps. Dans la présente étude, l'état asthmatique a été évalué longitudinalement tant au départ qu'au moment du contrôle. L'un des principaux atouts de notre étude est qu'elle nous a permis d'examiner les relations temporelles entre les facteurs de risque et les résultats cliniques et d'évaluer les enfants qui souffrent d'asthme persistant ou les enfants qui n'ont pas d'asthme au fil du temps, et de constater que les enfants atteints d'asthme persistant sont presque trois fois plus à risque d'être hospitalisés et quatre fois plus à risque d'utiliser les services de santé que ceux qui n'ont pas d'asthme. La nature longitudinale de l'enquête donne encore plus de poids à ces résultats car l'analyse intègre les facteurs de risques et les résultats au fil du temps tout en tenant compte de la corrélation intra-individus entre l'enquête de départ et l'enquête du contrôle.

Notre étude a démontré que les enfants des familles à faible revenu étaient exposés à un risque d'hospitalisation plus élevé au fil du temps peu importe leur état asthmatique, mais que leur USS était plus faible. Fait intéressant, d'autres études canadiennes ont donné lieu à des constatations similaires, particulièrement 
TABLEAU 1

Prévalence de l'hospitalisation et de l'utilisation des services de santé en fonction de l'état asthmatique

\begin{tabular}{|c|c|c|c|c|c|c|}
\hline & & & \multicolumn{2}{|c|}{ Hospitalisation } & \multicolumn{2}{|c|}{ Utilisation des services de santé } \\
\hline & & & $\begin{array}{c}\text { (Cycle 1) } \\
(\mathrm{n}=147800)\end{array}$ & $\begin{array}{c}\text { (Cycle 2) } \\
(\mathrm{n}=116000)\end{array}$ & $\begin{array}{c}\text { (Cycle 1) } \\
(\mathrm{n}=2562300)\end{array}$ & $\begin{array}{c}\text { (Cycle 2) } \\
(\mathrm{n}=2341300)\end{array}$ \\
\hline État asthmatique & $\mathbf{n}^{*}$ & $\%$ & $\%$ & $\%$ & $\%$ & $\%$ \\
\hline Enfants souffrant d'asthme persistant & 166600 & 5,33 & 16,77 & 8,29 & 95,27 & 91,45 \\
\hline Enfants nouvellement atteints d'asthme & 126000 & 4,03 & 6,07 & 5,84 & 89,84 & 92,94 \\
\hline Enfants souffrant d'asthme épisodique & 94600 & 3,02 & 9,41 & 4,75 & 93,78 & 76,72 \\
\hline Enfants ne souffrant pas d'asthme & 2741500 & 87,62 & 3,77 & 3,29 & 80,32 & 73,03 \\
\hline
\end{tabular}

* Le nombre d'enfants est pondéré et les valeurs sont arrondies à la centaine près. Nombre d'enfants non pondéré : 10148.

TABLEAU 2

Caractéristiques de la population à l'étude en fonction de l'état asthmatique

\begin{tabular}{|c|c|c|c|}
\hline & $\begin{array}{c}\text { Total } \\
(\mathrm{n}=2908100)^{*}\end{array}$ & $\begin{array}{l}\text { Asthme persistent } \\
\qquad(\mathrm{n}=166600)\end{array}$ & $\begin{array}{c}\text { Absence d'asthme } \\
\text { (n=2 } 741500)\end{array}$ \\
\hline Caractéristiques de base (cycle 1 ) & $\%$ & $\%$ & $\%$ \\
\hline \multicolumn{4}{|l|}{ Sexe } \\
\hline hommes & 50,54 & 62,58 & 49,81 \\
\hline femmes & 49,46 & 37,42 & 50,19 \\
\hline \multicolumn{4}{|l|}{ Âge (percentile) } \\
\hline de 1 à 4 ans & 37,87 & $27,62(4,18)$ & $38,50(95,82)$ \\
\hline de 5 à 8 ans & 35,14 & $40,46(6,60)$ & $34,81(93,40)$ \\
\hline de 9 à 11 ans & 26,99 & $31,92(6,78)$ & $26,69(93,22)$ \\
\hline Respiration sifflante chez l'enfant & 13,69 & 85,22 & 9,34 \\
\hline Enfants souffrant d'une allergie & 13,69 & 44,98 & 11,79 \\
\hline \multicolumn{4}{|l|}{ État de santé des enfants } \\
\hline excellent ou très bon & 90,06 & 61,01 & 91,82 \\
\hline bon, passable ou mauvais & 9,94 & 38,99 & 8,18 \\
\hline Faible adéquation du revenu & 9,77 & $6,94 \dagger$ & 9,94 \\
\hline Mère immigrante & 18,56 & $13,92 \dagger$ & 18,84 \\
\hline Mère biologique souffrant d'asthme & 4,78 & 15,45 & 4,14 \\
\hline Utilisation des services de santé & 81,18 & 95,27 & 80,32 \\
\hline Hospitalisations & 4,51 & $16,77 \dagger$ & 3,77 \\
\hline
\end{tabular}

* Le nombre d'enfants est pondéré et les valeurs sont arrondies à la centaine près. Nombre d'enfants non pondéré = 9462 .

Les pourcentages sont ajustés en fonction des données manquantes. Il est possible que le total ne soit pas 100, en raison de l'arrondissement de la valeur.

$†$ Le coefficient de variation se situe entre 16,6 \% et 33,3\%, ce qui est jugé marginal par Statistique Canada. 
TABLEAU 3

Rapports de cote ajustés de l'hospitalisation et de l'utilisation des services de santé en fonction de la régression logistique longitudinale*

\begin{tabular}{|c|c|c|c|c|c|c|c|c|}
\hline \multirow{3}{*}{$\begin{array}{l}\text { Facteurs de risque } \\
\text { Hommes }\end{array}$} & \multicolumn{4}{|c|}{ Hospitalisation } & \multicolumn{4}{|c|}{ Utilisation des services de santé } \\
\hline & \multirow{2}{*}{$\begin{array}{r}\text { RCA } \\
1,25\end{array}$} & \multicolumn{2}{|c|}{ IC de $95 \%$} & \multirow{2}{*}{$\frac{\text { Valeur prédictive } \dagger}{\mathrm{NE}}$} & \multirow{2}{*}{$\begin{array}{r}\text { RCA } \\
0,96\end{array}$} & \multicolumn{2}{|c|}{ IC de $95 \%$} & \multirow{2}{*}{$\frac{\text { Valeur prédictive } \dagger}{\mathrm{NE}}$} \\
\hline & & 0,99 & 1,57 & & & 0,85 & 1,08 & \\
\hline Asthme infantile & 2,52 & 1,71 & 3,70 & $<0,001$ & 3,80 & 2,69 & 5,37 & $<0,001$ \\
\hline Allergie infantile & 1,30 & 0,96 & 1,78 & NE & 1,61 & 1,33 & 1,94 & $<0,001$ \\
\hline Faible adéquation du revenu & 1,54 & 0,91 & 2,61 & NE & 0,58 & 0,44 & 0,76 & $<0,001$ \\
\hline Effet résiduel de la faible adéquation du revenu & 2,68 & 1,29 & 5,59 & 0,008 & 0,67 & 0,45 & 0,99 & 0,046 \\
\hline Mère immigrante & 0,65 & 0,43 & 0,98 & 0,039 & 1,06 & 0,88 & 1,28 & $\mathrm{NE}$ \\
\hline Cycle $2 \neq$ & 1,01 & 0,79 & 1,29 & NE & 0,87 & 0,78 & 0,98 & 0,019 \\
\hline
\end{tabular}

IC : intervalle de confiance; RCA : rapport de cote ajusté; NE = Négligeable (valeur prédictive inférieur à 0,05).

* $\quad$ En fonction d'un nombre non pondéré $=9462$.

$\dagger \quad$ En fonction de la régression logistique longitudinale et de facteurs de pondération où les poids normalisés sont utilisés.

‡ Temps contrôlé au cours des deux cycles.

en ce qui concerne l'hospitalisation. Une étude menée au Manitoba a révélé que les enfants âgés de 0 à 19 ans se situant dans le quintile de revenu le moins élevé risquaient trois fois plus d'être hospitalisés que ceux du quintile de revenu le plus élevé en $1999^{23}$. L'Institut canadien de la santé infantile a aussi fait état d'une tendance semblable relativement à l'hospitalisation chez les enfants en tenant compte du revenu du ménage ${ }^{24}$. Une étude récente portant sur les enfants nés dans une grande agglomération urbaine canadienne a conclu que la relation entre le désavantage socioéconomique et l'hospitalisation découlant de la nécessité de soins ambulatoires (l'asthme étant le diagnostic le plus fréquent) ainsi que l'hospitalisation toutes causes confondues était considérable, constante pour de nombreuses conditions, et stable au fil du temps jusqu'à l'âge de 9 ans $^{25}$. Étant donné que le Canada est doté d'un régime de soins de santé universel, ces différences ne peuvent s'expliquer par une difficulté d'accès aux soins de santé causées par une insuffisance de moyens financiers. Les facteurs qui font augmenter le risque d'un recours aux soins intensifs chez les enfants de la strate socioéconomique inférieure peuvent comprendre une plus forte prévalence de maladies, une plus grande sévérité des maladies, des comorbidités multiples, de mauvaises habitudes en matière de santé, le surpeuplement, des soins préventifs irréguliers, des problèmes de nutrition et une mauvaise forme physique ${ }^{25,26}$. De plus, des différences sur le plan économique quant à l'utilisation des corticostéroïdes à inhaler ont été notées chez les enfants asthmatiques $^{27-30}$. Bien que la présente étude ne renferme pas des renseignements détaillés sur l'utilisation des médicaments pour l'asthme, cette information aurait pu contribuer de façon significative aux différences observées dans les cas d'hospitalisations pour cause d'asthme et de problèmes liés à l'asthme, lesquels contribuent grandement à la morbidité générale au sein de la population d'asthmatiques ${ }^{31}$. Finalement, des facteurs liés au système de santé tels que la répartition du recours aux soins spécialisés peuvent aussi contribuer aux différences quant aux résultats des traitements. On estime qu'au Canada, la consultation d'omnipraticiens se répartit assez également selon les revenus en fonction des besoins. Cependant, les personnes qui ont un revenu supérieur sont, de façon significative, plus nombreux à solliciter des soins spécialisés que les personnes à revenu inférieur, et c'est pourquoi le taux global de consultation des médecins est quelque peu supérieur chez les mieux nantis ${ }^{32}$.

Notre conclusion quant à la répartition de l'asthme selon le statut socioéconomique (SSE) est aussi intéressante. Nous avons constaté que les enfants qui souffraient d'asthme persistant étaient moins susceptibles d'être issus de familles à faible revenu. Cela signifie que l'effet de l'asthme comme facteur de risque pour l'utilisation des soins de santé peut donner lieu à une confusion avec l'effet d'un revenu faible; cependant, ce biais potentiel a été réduit au minimum au moyen des ajustements de l'analyse multivariable. Les données précédentes sur la répartition de l'asthme selon le SSE amènent des résultats variés, et les conclusions diffèrent selon la définition de l'asthme et la méthodologie des études. Des enquêtes transversales réalisées aux États-Unis ont révélé qu'il y avait un lien entre le SSE et l'augmentation de la prévalence de l'asthme chez les enfants ${ }^{33,34}$. En Ontario, une étude longitudinale de la population fondée sur des données administratives en santé a montré que le revenu du quartier n'avait pas d'incidence sur la répartition de l'asthme persistant chez les enfants d'âge scolaire après correction des autres facteurs de risque ${ }^{35}$.

Il importe aussi d'examiner l'incidence du statut d'immigrant étant donné que le Canada a un taux d'immigration élevé et que la population immigrante peut être perçue comme vulnérable. Nos résultats ont révélé qu'une moins grande proportion d'enfants atteints d'asthme persistant étaient nés de mères immigrantes. Ces 
résultats correspondent à ceux des études menées aux États-Unis et ailleurs ${ }^{36-39}$ et confirment l'importance de l'immigration et de l'acculturation dans le développement de l'asthme et des allergies. Le caractère multifactoriel de l'immunoprotection, la sélection des immigrants en fonction de la santé et un biais de déclaration potentiel attribuable à la barrière linguistique et aux différences culturelles pourraient expliquer la prévalence moindre des cas déclarés d'asthme au sein de la population immigrante $e^{40-42}$. Notre étude a aussi révélé que les enfants de mères immigrantes sont hospitalisés en moins grands nombres. Cette constatation correspond à celle d'une récente étude menée par Quah et al. utilisant les données de l'Enquête sur la santé dans les collectivités canadiennes selon laquelle le nombre d'hospitalisations chez les minorités visibles, dont $81 \%$ étaient des immigrants, était moindre que chez les Canadiens blancs $^{43}$. Ce taux d'hospitalisation inférieur pourrait aussi s'expliquer en partie par le bon état de santé des immigrants, plutôt que par la difficulté d'accès, ce qui fait ressortir les caractéristiques uniques de la santé de ce groupe.

Certaines études canadiennes fondées sur des données administratives ont porté sur les cycles saisonniers des hospitalisations pour cause d'asthme dans la province de l'Ontario de 1988 à 200044,45. Les enfants âgés de 0 à 4 et de 5 à 9 ans enregistraient les plus forts taux d'hospitalisation. De plus, les hospitalisations des jeunes garçons étaient de deux à trois fois plus nombreuses que pour les filles du même âge ${ }^{44,45}$, une constatation qui correspond à nos résultats. Depuis le début des années 1990, on observe une tendance à la baisse quant au nombre total d'hospitalisations parmi la population générale, plus particulièrement chez les jeunes garçons $^{44,45}$. Notre étude a aussi fait ressortir que les hospitalisations chez les enfants atteints d'asthme persistant avaient presque diminué de moitié de 1994-1995 à 1996-1997 (16,8 \% c. 8,3\%). Chez les enfants qui ont participé aux deux cycles d'enquête, le pourcentage des hospitalisations en 1996-1997 a diminué par rapport à 1994-1995, tandis que le nombre d'enfants qui ont reçu un diagnostic d'asthme a augmenté durant la même période. Cette tendance peut s'expliquer en partie par le vieillissement de la cohorte entre les deux périodes, mais elle peut aussi traduire une amélioration du traitement de l'asthme et des soins de santé, laquelle aurait réduit le nombre total d'hospitalisations.

Notre étude avait certaines contraintes. D'abord, comme c'est le cas pour toutes les études fondées sur des données d'enquête, l'information sur l'utilisation des soins de santé a été obtenue des parents et comporte donc un biais de rappel. Il y avait un risque de sous-estimation du nombre d'hospitalisations étant donné qu'un enfant qui avait été hospitalisé à plusieurs reprises n'était compté qu'une seule fois par année; de plus, l'hospitalisation était définie comme une admission pour quelque motif que ce soit et non seulement pour des maladies respiratoires. Cependant, la constance de la tendance à la baisse du nombre d'hospitalisations observée au cours de notre étude et de la précédente confirme nos conclusions. L'USS a été déterminée en fonction de la question suivante : "L'an dernier, combien de fois avez-vous vu un médecin ou parlé avec lui au sujet de la santé de votre enfant? ». Cette question peut fausser le nombre de consultations médicales, car les répondants pourraient compter deux fois le nombre de visites ou d'appels téléphoniques effectués le même jour et exclure les visites d'urgence dans les hôpitaux. Ainsi, nous avons décidé de dichotomiser les résultats de l'USS afin de réduire le biais potentiel, et cela peut donner lieu à une sous-estimation du nombre total de visites chez le médecin. Globalement, ces contraintes quant à la déclaration des variables de l'USS peuvent réduire les différences détectables dans l'USS au sein de la population, et c'est pourquoi il est peu probable que le fardeau attribuable à l'asthme ait été surestimé. Une autre contrainte de notre étude a trait au manque d'information sur la gravité de l'asthme. Comme certaines données révèlent que les personnes atteintes des formes d'asthme les plus graves affichent des taux de morbidité et d'USS les plus élevés, il est possible que l'accroissement de l'USS soit surtout attribuable aux formes d'asthme les plus graves ${ }^{46-49}$.

\section{Conclusions}

Notre étude longitudinale de la population confirme que l'asthme est lié de façon significative à des taux d'hospitalisation et d'USS supérieurs chez la population pédiatrique, et confirme par ailleurs que, chez les enfants de familles à faible revenu, le taux d'hospitalisation est plus élevé et l'USS est plus faible. Les programmes qui visent à réduire le fardeau économique des hospitalisations pédiatriques doivent par conséquent se concentrer sur l'asthme et les populations à faible revenu.

\section{Remerciements}

La réalisation de ce projet a été financée par l'institut de recherche de l'Hospital for Sick Children, Toronto (Ontario), Canada. La $\mathrm{D}^{\mathrm{re}}$ Teresa To est subventionnée par l'Université de Toronto (Life Sciences Committee, Prix Dales en recherche médicale en 2007).

\section{Avertissement}

Cette analyse a été fondée sur le fichier maître de Statistique Canada Enquête longitudinale nationale sur les enfants et les jeunes qui contient des données anonymisées recueillies dans le cadre de l'enquête spéciale de 1994-1995 et de 1996-1997. Tous les calculs ont été réalisés par le département des Sciences de l'évaluation de la santé des enfants de l'Institut de recherche de l'Hospital for Sick Children, et la responsabilité de l'utilisation et de l'interprétation de ces données incombe uniquement aux auteurs. Les opinions exprimées ne représentent pas nécessairement celles de Statistique Canada. 


\section{Références}

1. Casanova C, Starfield BJ. Hospitalizations of children and access to primary care: A cross-sectional comparison. Int $\mathrm{J}$ Health Serv. 1995;25:283-94.

2. Gadomski A, Jenkins P, Nichols M. Impact of a medicaid primary care provider and preventive care on pediatric hospitalization. Pediatrics. 1998;101(3):1-10.

3. Flores G, Abreu M, Chaisson CE, Sun D. Keeping children out of hospitals: parents' and physicians' perspectives on how pediatric hospitalizations for ambulatory sensitive conditions can be avoided. Pediatrics. 2003;112:1021-30.

4. Flores G, Abreu M, Tomany-Korman S, Meure J. Keeping children with asthma out of hospitals: parents' and physicians' perspectives on how pediatric asthma hospitalizations can be prevented. Pediatrics. 2005;116:957-65.

5. Brownson RC, Remington PL, Davis JR. Chronic disease epidemiology and control. $2^{\text {nd }}$ ed. Washington (D.C.): American Public Health Association; 1998.

6. Santé Canada. Le fardeau économique de la maladie au Canada, Ottawa, Santé Canada, 1998. Rapport nº : ISBN 0-662-88050-1.

7. To T, Ungar W. Outpatient health services use by children in Ontario, Toronto, Institute of Clinical Evaluative Studies, 2001. Rapport $\mathrm{n}^{\circ}$ : ISBN 0-9686318-6-X.

8. Ungar WJ, Coyte PC, Pharmacy Medication Monitory Program Advisory Board. Prospective study of the patient-level cost of asthma care in children. Pediatr Pulmonol. 2001;32:101-8.

9. Santé Canada. Les maladies respiratoires au Canada, Ottawa, Santé Canada, 2001. Rapport $\mathrm{n}^{\circ}$ : ISBN 0-662-86162-0.

10. To T, Dell S, Dick P, et al. Burden of childhood asthma [Internet], Toronto, Institute for Clinical Evaluative Sciences, 2004 mai. URL : http://www.ices.on.ca/ file/ACF77.pdf.
11. Ressources humaines et Développement des compétences Canada, Statistique Canada. Guide de l'utilisateur des microdonnées : Enquête longitudinale nationale sur les enfants et les jeunes. Cycle $1,2^{\text {e }}$ éd., Ottawa, ministre de l’Industrie, 1997.

12. Ressources humaines et Développement des compétences Canada, Statistique Canada. Guide de l'utilisateur des microdonnées : Enquête longitudinale nationale sur les enfants et les jeunes. Cycle 1, $3^{\text {e }}$ éd., Ottawa, ministre de l'Industrie, 1999.

13. To T, Vydykhan $\mathrm{T}$, Dell S. Is obesity associated with asthma in young children? J Pediatr. 2004;144:162-8.

14. Diggle PJ, Liang KY, Zeger SL. Analysis of longitudinal data, Oxford, Claredon Press, 1994. Chapitre 8, Marginal models, p. 14668.

15. Carey V, Zeger SL, Diggle P. Modelling multivariate binary data with alternating logistic regressions. Biometrika. 1993; $80(3): 517-26$.

16. Lipsitz SR, Fitzmaurice GM, Orav EJ, Laird NM. Performance of generalized estimating equations in practical situations. Biometrics. 1994;50:270-8.

17. Stokes ME, Davis CS, Koch GG. Categorical Data Analysis: using the SAS system, $1^{\text {ière }}$ éd., Cary (NC), SAS Publishing, 1995. Chapitre 15, Advanced topic: The generalized estimating equation (GEE) method, p. 413-23.

18. Statistique Canada. Les seuils de faible revenu. Ottawa, Statistique Canada, 1996.

19. Hosmer DW, Lemeshow S. Applied logistic regression, New York, John Wiley and Sons, Inc., 1989.

20. To T, Cadarette SM, Liu Y. Biological, social, and environmental correlates of preschool development. Child Care Health Dev. 2001; 27(2):187-200.
21. SAS Institute Inc. SAS/STAT user's guide, version 8. Cary (NC), STATS Publishing Inc., 1999:1375-428.

22. Chen Y, Dales R, Krewski D. Asthma and the risk of hospitalization in Canada: the role of socioeconomic and demographic factors. Chest. 2001;119(3):708-13.

23. Brownell M, Martens P, Kozyrskyj A, et al. Assessing the health of children in Manitoba: a population-based study, Winnipeg (Manitoba), Manitoba Centre for Health Policy, 2001.

24. Institut canadien de la santé infantile. The health of Canada's children: a $\mathrm{CICH}$ profile, Ottawa, Institut canadien de la santé infantile, 1994.

25. Agha MM, Glazier RH, Guttmann A. Relationship between social inequalities and ambulatory care-sensitive hospitalizations persists for up to 9 years among children born in a major Canadian urban center. Ambul Pediatr. 2007;7(3):258-62.

26. Sin DD, Svenson LW, Cowie RL, Man SF. Can universal access to health care eliminate health inequities between children of poor and nonpoor families? A case study of childhood asthma in Alberta. Chest. 2003;124(1):51-6.

27. Kozyrskyj AL, Mustard CA, Simons FE. Inhaled corticosteroids in childhood asthma: income differences in use. Pediatr Pulmonol. 2003;36(3):241-7.

28. Kozyrskyj AL, Mustard CA, Simons FE. Socioeconomic status, drug insurance benefits, and new prescriptions for inhaled corticosteroids in schoolchildren with asthma. Arch Pediatr Adolesc Med. 2001;155(11):1219-24.

29. Ungar WJ, Kozyrskyj A, Paterson M, Ahmad F. Effect of cost-sharing on use of asthma medication in children. Arch Pediatr Adolesc Med. 2008;162(2):104-10.

30. Blais L, Beauchesne MF, Levesque S. Socioeconomic status and medication prescription patterns in pediatric asthma in Canada. J Adolesc Health. 2006;38(5):607 e9-16. 
31. To T, Gershon A, Tassoudji M, et al. The burden of asthma in Ontario, Toronto (Ontario), Institute for Clinical Evaluative Sciences, 2006.

32. van Doorslaer E, Masseria C, Koolman X Inequalities in access to medical care by income in developed countries. CMAJ. 2006;174(2):177-83.

33. Halfon N, Newacheck PW. Childhood asthma and poverty: differential impacts and utilization of health services. Pediatrics. 1993;91(1):56-61.

34. Higgins PS, Wakefield D, Cloutier MM. Risk factors for asthma and asthma severity in nonurban children in Connecticut. Chest. 2005;128(6):3846-53.

35. To T, Gershon A, Wang C, Dell S, Cicutto L. Persistence and remission in childhood asthma - a population-based asthma birth cohort study. Arch Pediatr Adolesc Med. 2007;161(12):1197-204.

36. Eldeirawi K, McConnell R, Freels $\mathrm{S}$, Persky VW. Associations of place of birth with asthma and wheezing in Mexican American children. J Allergy Clin Immunol. 2005;116(1):42-8.

37. Hjern A, Haglund B, Bremberg S, RingbackWeitoft G. Social adversity, migration and hospital admissions for childhood asthma in Sweden. Acta Paediatr. 1999;88(10): 1107-12.

38. Netuveli G, Hurwitz B, Sheikh A. Ethnic variations in incidence of asthma episodes in England \& Wales: national study of 502,482 patients in primary care. Respir Res. 2005;6:120.
39. Gibson PG, Henry RL, Shah S, Powell H, Wang H. Migration to a western country increases asthma symptoms but not eosinophilic airway inflammation. Pediatr Pulmonol. 2003;36(3):209-15.

40. Gold DR, Acevedo-Garcia D. Immigration to the United States and acculturation as risk factors for asthma and allergy. J. Allergy Clin. Immunol. 2005;116(1):38-41.

41. Kalyoncu AF. Symptoms of asthma, bronchial responsiveness and atopy in immigrants and emigrants in Europe. Eur Respir J. 2002;19(5):980-81.

42. Choudhry S, Seibold MA, Borrell LN, et al. Dissecting complex diseases in complex populations: asthma in Latino Americans. Proc Am Thorac Soc. 2007;4(3):226-33.

43. Quan H, Fong A, De Coster C, et al. Variation in health services utilization among ethnic populations. CMAJ. 2006:174:787-91.

44. Crighton EJ, Mamdani MM, Upshur RE A population based time series analysis of asthma hospitalizations in Ontario, Canada: 1998 to 2000. BMC Health Serv Res. 2001:1-7.

45. To T, Guttmann A, Dick P. Inpatient and day surgery use by children in Ontario, Toronto, Institute for Clinical Evaluative Sciences, 2001. Rapport $n^{\circ}$ : 0-9699405-9-9.

46. Van Ganse E, Antonicelli L, Zhang Q, et al. Asthma-related resource use and cost by GINA classification of severity in three European countries. Respir Med. 2005;100:140-47.
47. Bootman JL, Crown WH, Luskin AT. Clinical and economic effects of suboptimally controlled asthma. Manag Care Interface. 2004;17(1):31-6.

48. Berggren F, Hjelmgren J, Lindgren B. Differences in health care utilisation and workdays lost between individuals with and without asthma. Appl Health Econ Health Policy. 2003;2(3):165-70.

49. Halterman JS, Yoos HL, Sidora K, Kitzman $\mathrm{H}$, McMullen A. Medication use and health care contacts among symptomatic children with asthma. Ambul Pediatr. 2001;1(5):275-9. 